\title{
Ecological Counseling Model to Reduce Parenting Stress on Multiple Disabilities and A Visual Impairment (MDVI) Parents
}

\author{
Sunardi \\ Universitas Pendidikan Indonesia \\ Bandung, Indonesia
}

\begin{abstract}
This study aims are to formulate and test the effectiveness of ecological counseling models to reduce stress parenting by parents of children with Multiple Disabilities and a Visual Impairment (MDVI). The study was conducted in P2TLV YPWG Bandung through a qualitative approach with case study methods on 7 cases and carried out in the context of early intervention. The results show that the parenting stress of their experience is quite complex and diverse. Diversity occurs both in symptoms, forms, sources, and dynamics. The results of the implementation of the ecological counseling model which was built through group counseling, with the formation of parent group support and home visits as the main pillar, and in the process prioritizing the pattern of collective empowerment, coping strategies, and attachments implemented in the context of early intervention, showed the model was able to reduce parenting stress on MDVI parents. This is marked by the occurrence of behavioral changes in a more positive direction than the implementation of the model before. Thus, this approach deserves to be disseminated and used as one of the references in overcoming parenting stress in parents of MDVI children.
\end{abstract}

Keywords - stress parenting; MDVI; children with special needs; ecological counseling.

\section{INTRODUCTION}

Every parent is not only hopes his child is born normal, but also perfect or a truly special child or "super baby" [1]. However, the fact is not always the case. Many of them have children with special needs, including children with Multiple Disabilities and A Visual Impairment (MDVI). If this happens, the dream of having a child that is "super baby" can quickly disappear and change with disappointment, sadness, and suffering.

Being an MDVI parent is also not an option, therefore there are no parents who are ready to become parents of the MDVI children. If this happens, it can be a stressful psychological experience and then create a variety of psychological crises. First, the crisis of symbolic death, namely the destruction of dreams for the coveted child. Second, the attitude of rejection, which is characterized by symptoms of "doctor shopping", which become part of the parents' refusal of a previous diagnosis or an attempt to get a miracle. Third, parenting stress, which is a crisis related to the emergence of anxiety and tension in care, and child education.
The above conditions, ultimately can lead to the existence of rejection, powerlessness, neglect, high-level tension and disputes, or even in many cases end in divorce. This is the reason why there is a known of "disability and divorce" syndrome. If the occurrence of stress parenting on parents cannot be managed properly it can have an impact on the role of parents' dysfunction. It leads to poor parenting behavior, and a decrease in the levels of psychological well-being, affection, social, and economic. If this happens continuously, it will make the family fragile, not resilient, which in the end will not only harm itself but also the optimal development of the child.

Responding to the emergence of parenting stress symptoms in MDVI parents, it requires the care of the counselor to help them. Counseling can be an effective way for couples who have children with special needs in order to restore balance [2]. It is a way to talk about their hopes, dreams, and daily fear and frustration openly so as to reduce isolation and their anger, and can provide more benefits in facilitating their adjustment, rather than direct therapy or remediation in children with special needs [3].

It should be understood that the main source of stress stressors faced by children with special needs parents is the condition of their children. For this reason, special and early intervention is needed for their children to minimize learning barriers and the developmental obstacles they face, and services that are considered appropriate for that are early intervention services.

The essence of early intervention is to help children and their families. This means that the targets of early intervention are both for children and also their families. Families must be an integral part of the entire process and the implementation of early intervention.

In Indonesia, even though early intervention services are new, they have developed quite rapidly. The problem, infield practice has not fully embraced the new paradigm in early intervention, namely by making parents the center. As a result, the services provided are not optimal for child development.

According to Rochyadi (interview on June 10, 2016), the objective conditions in the field showed the real problems faced by therapists were not only in children, but also in parents, namely their anxiety when seeing their children was different from normal children in general. Parents tend to send 
their children to the clinic after reaching school age. As a result, therapists tend to face heavy or increasingly complex children with special needs clients. On the other hand, parents want the handling of the problems faced by their children is done immediately and securely, making it difficult for the therapist. If it is done early, it is believed that handling will be easier. Because of that, from the therapist point of view, what is more difficult is how to deal with parents, not their children.

One of the early intervention agencies for people with low vision and Multiple Disabilities Visual Impairment (MDVI) in West Java is the Low Vision Integrated Service Center (P2TLV) of the Bandung Wiyata Guna Foundation (YPWG). Besides doing direct service to low vision clients, one type of service provided at this institution, namely guidance and counseling to parents and their families. The problem is, because of the absence of counselors, the guidance and counseling program have not yet done well.

In the view of ecological theory, human behavior is the result of its social context, the result of the dynamic interaction process between children and their environment, especially the family as a system. Therefore, the family is the most crucial ecology of human development. Family is a "critical system" where children learn how to satisfy their needs and how to deal with the world.

Based on the above, in the context of early intervention, the counseling approach which is considered effective to reduce parenting stress in children with special needs parents is an ecological approach. Through the application of ecological counseling, parenting stress issues experienced by children with special needs parents are conceptualized by integrating personal and environmental factors with a focus of improving the quality of their interactions towards achieving a harmonious and satisfying life. Especially through the creation of a learning environment that was deliberately designed and developed to facilitate learning through the provision of support structures, opportunities and rewards.

Based on the above problems, this study intends to find ecological counseling models that are considered appropriate and effective to reduce parenting stress faced by children with special needs parents in the setting of early intervention. The discovery of the model is considered important if the parenting stress problems faced by children with special needs parents are not immediately helped, not only will it have a negative impact on their mental health but also can give a negative impact on care for their children who are special needs children, which in turn can harm various developments. for children with children with special needs, with research questions: (1) What is the profile of parenting stress faced by MDVI parents? (2) What is the ecological counseling model which can reduce parenting stress in MDVI parents?.

\section{METHOD}

This study aims to formulate an ecological counseling model in the context of early intervention that is able to reduce parenting stress in parents who have MDVI children. To achieve this goal, the research was carried out through a qualitative approach with a case study method of seven parents who had early MDVI children (less than five years) and were participating in an early intervention program in P2TLVYPWG Bandung. Data collection techniques are carried out through interviews, observation, and documentation studies. Data analysis was carried out using a framework which consisted of three phases, namely data reduction, data display and drawing conclusions and verification.

In general, this research was carried out in 3 stages. In the first stage, an in-depth study of seven cases were conducted to obtain the parenting stress profile experienced, including the form, nature, impact, and their needs. The second stage formulates a model that is considered appropriate to reduce parenting stress faced. Meanwhile, in the third stage is the implementation of the model to determine its effectiveness.

\section{RESULT AND DISCUSSION}

The results of a study of seven parents who had MDVI children showed that the presence of children with special needs in the midst of families tended to give birth to a variety of problems which then influenced changes in their family dynamics, both internally and externally. These problems include primary and secondary problems. Primary problems are problems that are directly faced by parents towards the presence of their children, such as shock, disappointment, fear, and rejection. Whereas secondary problems are problems that arise later related to how to care for, educate, care for them, and raise them. In other words, parents who have MDVI children tend to be faced with problems related to parenting stress. This confirms that the presence of MDVI children in the midst of families tends to give birth to a variety of psychological and social crises for parents and their families, namely the destruction of the desired aspirations for children, as well as their relation to guidance, education and care.

The results of the study of seven cases who were MDVI children with special needs parents in P2TLV Wiyata Guna Foundation Bandung, namely MM-CS, MM-RH, MM-FN, MM-DF, MM-IB, MM-NT, and MM-RD. With the focus of research is parenting stress, which includes psychological, social, physical, and parent-child interactions displayed. In short, the results can be explained that the parenting stress that occurs in MDVI parents in P2TLV YPWG Bandung is quite complex and diverse. Diversity occurs in symptoms, forms, sources and dynamics. Besides that, even though the symptoms of stress parenting which are shown for each research subject is characteristic. There are some general symptoms of parenting stress in children with special needs parents, including:

- Sad because his son did not show significant progress.

- Worried that the condition of his child could get worse, his future was uncertain, would later become an environmental burden, or become a victim of environmental injustice.

- Afraid that his child will suffer from being a victim of the environment used as mockery, harassed, shunned, or rejected by the social environment. 
- Worry not be able to treat his child properly, because he feels he does not have special skills according to the condition of his child.

- Worrying about the environment, including his closest environment (siblings) will not care, cannot love, and protect it.

- Fear of the environment being unfair by giving stigma or a negative reaction to themselves and their children, so that they tend to withdraw or limit themselves in association, or at least be very careful in expanding their relationships.

- Often feeling confused, frustrated and then afraid of losing patience and emotions are not distracted.

- Anxiety about education, relationships, and the future of his child.

- Feelings that the environment does not always support them and their children, so they tend to be unwilling or avoid being in a public place.

- Emergence of anxiety that their children can be threaten the integrity of their household.

- Physical exhaustion, because it requires more attention, time, and energy. The time is spent more on his child.

- Pessimistic about uncertainty about the future education of their children.

- Lack of confidence, cold and quiet. His attitude is closed and not excited.

- Pessimistic about his child's condition and confused about not knowing what to do.

- Confusing in choosing and determining the right school or place of education for their children.

Based on the above conditions, basically MDVI parents need the following:

- An environment that can understand, accept, and respect themselves and their children without conditions.

- An environment that is truly capable of supporting themselves and their children, so that they can feel safe and comfortable in them.

- An environment that is able to provide convenience for the progress of learning and the development of their children.

- An environment that is able to provide information and skills improvement needed to improve the quality of his caring.

- An environment that allows mutual sharing and mutual support between one another.

- Learning environment that can build self-confidence and optimism for him.
- An environment that is capable of being empathic and guarantees that it will not hurt itself and their children.

- An environment that is able to make them as resilient parents: have a strong, strong, and strong in dealing with various problems.

- The environment that is directly capable of helping the progress and positive development of children with special needs.

The above research findings are then used as an empirical basis for the preparation of ecological counseling models that will be tested for effectiveness. Model Development Results Development of ecological counseling models to reduce stress parenting in MDVI parents in the setting of early intervention has been done through these steps: (1) preliminary study, which includes empirical studies through case studies and theoretical studies through literature study, (2) formulation hypothetical model, (3) expert judgment, (4) formulation of operational model, (5) model effectiveness test, (6) model a dissemination.

\section{A. Hypothetical Model}

Hypothetical model of ecological counseling, based on two bases, namely the actual basis of the results of case studies and a conceptual basis based on literature study. The proposed model framework includes: (1) introduction, (2) empirical and conceptual basis for model building, (3) model orientation, (4) vision, mission, and objectives, (5) program, (6) counseling stages, and (7) the role of counselor. After expert judgment is done with the intention to validate the contents of the hypothetical model that has been made, the results are:

- In general, the hypothetical model that is formulated is good enough, but to be used as an operational model it needs additional or more technical and operational explanations.

- The format or structure of the model being developed should refer to one model that has been considered established.

- Need to add other components that are considered important, namely: (a) procedures or stages of counseling, (b) evaluation and indicators of success of counseling, (c) objectives, and (d) counselor competence. Or adjust to the model used as a reference.

- To be easily understood, and not to contain meaning or multiple interpretations, it is necessary to pay attention to the accuracy of the language. For this reason, language needs to be simplified, so that it is more concise, clear and firm.

Based on these inputs, the researcher then revised the structure and content of the model that had been formulated beforehand and the results were then consulted and reconfirmed to each weigher to be agreed upon as an operational model that would be empirically tested for effectiveness. 


\section{B. Operational Model}

Based on the results of the validation of the theoretical model that has been carried out, the Ecological Counseling operational model is to reduce parenting stress in children with special needs parents in the context of this early intervention, as follows:

- Determination of the model component in the preparation of this ecological counseling model, refers to the model of The Texas Comprehensive, Developmental Guidance and Counseling Model, which consists of six components, namely: (1) Introduction, (2) Model Structure, (3) Responsibility and Counselor Competence, (4) Scope and Sequence, (5) Model Implementation Process, and (6) Model Evaluation and Success Indicators.

- In term of the method or technique used, this ecological approach model uses three main methods, namely group counseling, parent support groups, and home visits as the main pillar. In group counseling, the practice is based on the integrative approach proposed by Corey $\&$ Corey [4] by combining three domains (the cognitive domain, the affective domain, and the psychomotor domain) as the basis for counseling practice. Group counseling is chosen as an approach to help reduce parenting stress faced by children with special needs parents, given that group counseling focuses on the interpersonal relationship process and emphasizes problem solving strategies that emphasize awareness, feelings, and behavior. Use interactive feedback and support methods in the frame here and now (in here and now time frame). The function of the counselor is more as a facilitator. In accordance with the concept of learning ecology, this model emphasizes the need for parents to develop opportunities, support, rewards, and ease for parents to learn to build balanced, positive and healthy interactions by placing sharing activities, coping strategies, child exchange, and attachments as main concern.

- Judging from the pattern of the counselor's relationship with parents, this ecological counseling model is built based on the pattern of the collective empowerment relationship.

The choice of the collective empowerment relationship model is based on the reason that the model is also the most advanced model of counseling that reflects the implementation of the views, ecological view. The essence of the collective empowerment model is the synergy of strength that is built from strength collaboration and then synergistically radiates community ecology. In the collective empowerment model, strength is not only obtained by all participants through partnership, but naturally the power produced is also transformed. Thus, this strength can be a capacity building, with participants gaining competence, ability, obtaining resources, and capabilities without taking some strength from others. In this model, the capacity is also a source that is not limited to everyone in partnership.
In the application of early intervention, the collective empowerment model focuses on: (1) family centrality, (2) family choice as a basis for decision making, as well as family strength and capability as a focus of intervention. This model also focuses on three important elements, namely: (1) access to resources, (2) participation, and (3) changing community ecology. The element of access to resources are: (1) participants must know about substantially different sources, and (2) participate in the decision-making process. In this context, the professional or counselor's role is to facilitate or guide the family in obtaining sources, both found in the formal and informal systems. For this reason, families are encouraged to be able to build networks with family members, friends, neighbors, and communities in obtaining resources in their environment. In second element, participation, collective empowerment means giving families the opportunity to participate in decision-making, with equal power sharing, not subordinate or hierarchical. The choice of family participation can vary in level, time, and way. It makes this model choices and participation in decision making are more determined by the family than the counselor. As for the third element, changes in community ecology, this parent's counseling model assumes that the mother is a "broken" person and needs to be "matched" in the mother-child microsystem relationship.

In the collective empowerment model, the role of counselors or more professionals as facilitators, collaborators, or partners rather than as experts or specialists, is therefore required to have a desire to share, as well as an open, trusting, respectful, and caring attitude. The expected results from this collective empowerment model are synergy, the creation of new sources, and the satisfaction of participants. Synergy, can only be awakened if parents' feelings are appreciated by the counselor and the counselor's feelings are also appreciated by parents, as well as collaboration between both.

Fourth, in terms of the stages of counseling, this model was built on the basis of the stages of counseling [5] who have described the six stages of the MDVI family eclectic counseling model. To move from stage one to another stage is flexible and can change with focus on six stages, and can return to the previous stage if needed. The six stages are:

- Stage 1 Establish trust relationships

- Stage 2 Verbalization of emotions and factual aspects of client problems

- Stage 3 Identify alternative actions

- Stage 4 Evaluation of alternative actions

- Stage 5 Commitment to the action plan

- Stage 6 Summing up progress and evaluating the level of achievement of objectives and follow-up.

Fifth, reviewed from the developed service format, this ecological counseling model was also built with reference to the opinion of [6] which concluded that the technical implementation of counseling for children with special needs parents should be carried out primarily through group counseling, with the reason it is cheaper and allows mutual 
support. While individual counseling or family counseling are a follow-up service. The meeting session can be divided into two parts, the psychoeducation session and support group. In implementation part it should be flexible. It can be done through face-to-face, home visits, e-mail, or by telephone. Formulation of the operational model as referred to above (complete attached).

\section{Model Effectiveness}

The results of the qualitative effectiveness of the model were carried out by describing prominent changes in behavior in each research subject. By looking at the changes that occur in each research subject that occurs and emerges, it is believed to be the result of the application of the applied ecological counseling model. It can be concluded that the ecological counseling model which is applied to children with special needs parents can reduce the parenting stress they face.

In detail, some of the behavioral changes that believed to be an indicator of the effectiveness of the counseling model applied are:

- Able to change behavior from a closed attitude to a more open attitude, both open to self-experience and in accepting other people's experiences.

- Able to change the attitude of lack of confidence, quiet, withdrawn to a more daring, confident, familiar and expressive behavior.

- Able to change behavior from feeling isolated, feeling alone, and less valuable to feelings which are more acceptable, accompanied, and valued.

- Able to change attitudes that were initially pessimistic and lacking in enthusiasm to be more optimistic and enthusiastic.

- Able to change attitudes and feelings that are oriented inward to attitudes and feelings that are oriented outward.

- Able to reduce concerns about education and the future of their children.

- Able to change the perception that the environment is perceived as lacking in understanding, accepting, and appreciating the understanding that the environment can be a supportive media that cares.

- Able to place parents in certain positions that are more respectable and needed by others.

- Able to build awareness to help and help each other.

The above changes were also strengthened by P2TLV YPWG responsible person who was aware or not often stated to the researchers that parents now seemed more enthusiastic and diligent in taking part in the therapy activities.

The success of ecological counseling models in reducing stress parenting in MDVI parents shows that this counseling is able to build parents' awareness and understanding and also to bring changes in attitudes and behavior towards a more positive attitude. This reinforces the assumption that the success of parents in overcoming the psychological crisis as a result of the presence of their disabled children in the midst of their families is really dependent on the information obtained, tend to move from negative to positive, from shock, recognition, rejection and acceptance accompanied by constructive activities. The success of parents in the pattern of response is very dependent on information and guidance obtained from people who are experts in their fields.

The sensitivity of parents in capturing the obstacles and special needs of MDVI children in the early stages of development, are the entry points for parents to build maximum quality of development. However, this will not happen if the sensitivity is not followed by special efforts to overcome the obstacles to learning and the development of their children.

Ecological counseling model that emphasizes group counseling strategies, with the application of parent group support techniques, groups of child exchange and attachments in this research are essentially efforts to strengthen gratitude, compassion, and reasonable protection for their children. This is important because according to Dunst and Trivette [7] that the behavior and development of children with special needs are strongly influenced by their families, especially by the dimensions of health and well-being of parents, family functioning, and the style of interaction between parents and children. While the functioning of each (parents, family, and children) is very dependent on the role of experts in carrying out their functions as "social support".

One aspect of parenting stress that has not been reduced through the mood of ecological counseling is related to the role satisfaction as MDVI parents. This fact is actually something reasonable, understandable, and with various considerations can be accepted. First, the satisfaction problem is a problem that is only met if the needs or expectations are achieved. One of the parents' greatest hopes for their children is MDVI is "curable". The problem is, what happens to the child is a condition, not an illness. So, it can never be cured, because there is no cure medically.

Second, for people who are depressed, experiencing constant disappointment will make it difficult for them to feel satisfied. Being less sensitive in responding to the child's progress, and the pattern of relationships which build up later becomes very challenging, hostile, and problematic. When that happens, according to Crnic \& Low[8] children's behavior and the relationship of parents and children will deteriorate. In the Parent-Child-relationship theory, shows that pressure can have an effect not only on parents and their behavior, but also on children and their relationships with parents. While Dubbs [8] asserts that when parents experience continuous pressure, parents will experience a change in their role. They will have difficulties in completing their dilemmas and experience a decrease in satisfaction with their roles.

\section{CONCLUSION}

Based on the findings of the study and discussion, it can be concluded that an effective ecological counseling model to reduce parenting stress in MDVI parents in the early intervention setting is a counseling model which places the 
importance of environmental management or engineering which is able to provide opportunities, support, rewards, and facilities for clients in learning to build balanced, positive and healthy interactions by placing sharing, coping stress, child exchange, and attachments as the main concern, as well as making group counseling, parent support groups, and home visits as the main pillars. The implementation has been done through differentiated services, integrated with intervention services, built in a multidisciplinary work team with the role of expert teams as social support, counselor roles and responsibilities as facilitators as well as the spearhead of multidisciplinary work teams, as well as placing the vision of collective empowerment as a relationship pattern counselor with parents.

The effectiveness of this model is characterized by attitudinal and behavioral changes from being initially negative to be a more positive direction, such as: from an attitude that tends to be closed to a more open attitude; from a lack of confidence, quiet, withdrawing to a more daring, confident, familiar and expressive behavior; from feeling isolated, feeling alone, fragile, and less valuable to feelings that are more acceptable and accompanied, and stronger and more valued; from being pessimistic and lacking in enthusiasm to being more optimistic and enthusiastic.

\section{REFERENCES}

[1] N.H. Fallen, and W. Umansky, Young children with special needs, Columbus-Ohio: Charles E Merrill Publishing Company, 1985.

[2] K. Putz, For better or worse, and kids with disabilities, 2010.

[3] D. A. Henderson and C. L. Thompson, Counseling children, Cengage Learning, 2010.

[4] M.S. Corey and G. Corey, Process and practice groups, London: Thomson Brooks/Cole, 2006.

[5] M. E. Ziolko, "Counseling parents of children with disabilities: A review of the literature and implications for practice," Journal of Rehabilitation, vol 57, no 2, pp.29, 1991

[6] C. Hansley, Support system for parent of children with special Needs, Eastem Michigan University, 2007.

[7] K. Marfo, Parent-Child interaction and developmental abilities: theory, research, and intervention, New York: Praeger, 1988.

[8] J. L. Dubbs, Parent stress reduction through a psychosocial intervention for children diagnosed with attention-deficit/hyperactivity disorder, Pennsylvania: Indiana University of Pennsylvania, 2008 